\title{
Study on "Internet +" intelligent environmental protection to promote the modernization of environmental governance in Liaoning Province
}

\author{
Qian Kun ${ }^{1, *}$, Yang Xiao Nan² \\ ${ }^{1}$ Dalian Polytechnic University, Department of Business Administration, Dalian, Liaoning Province, China \\ ${ }^{2}$ Dalian Polytechnic University, Department of Business Administration, Dalian, Liaoning Province, China
}

\begin{abstract}
Through the study of urban environment governance in liaoning province, analyzing the present status quo and problems of environmental governance, we hope to solve the problems of urban environment governance in liaoning province, and put forward Suggestions on promoting the modernization of urban environmental governance.Then building intelligent monitoring and early warning system, intelligent environmental monitoring system and intelligent service system,in order to enhance the modernization level of urban environmental governance in Liaoning Province.
\end{abstract}

\section{The introduction}

With the continuous development of digital technologies such as artificial intelligence and big data, urban governance also pays more attention to realizing urban management through digital operation, so as to realize the innovation of urban governance mode and concept. The transformation from digitization to intelligence and wisdom has become the mainstream trend of accelerating the construction of urban governance system and realizing the modernization of urban governance, which is a problem that must be considered in urban renewal.

The urbanization construction in Liaoning Province is still at a low level, but the problems of urban environmental governance are still significant. The main reasons are that the urban governance system is not perfect, the urban environmental governance is weak, and the environmental monitoring is difficult. The application of "Internet + " intelligent environmental protection can link data resources related to the urban environment with specific urban problems. Through real-time monitoring of urban environmental data, emergency response plans can be formulated to improve the efficiency and quality of environmental governance. Through investigation and analysis, the application of intelligent environmental protection system in urban environmental governance can improve the status quo of environmental governance in Liaoning Province. Therefore, it is necessary to conduct in-depth research on "Internet + " intelligent environmental protection.

\section{The concept and connotation of "Internet +" intelligent environmental protection}

\subsection{The concept of "Internet +"}

"Internet +" refers to the integration of Internet technology and other traditional industries. Through the use of Internet-related technologies, it can open up new markets for traditional industries, discover new opportunities, and give full play to the advantages of traditional industries. "Internet + " is the best choice for traditional industries to adapt to The Times, meet the challenges and realize optimal transformation, and it is an important force to promote the continuous development of society and economy.

\subsection{The concept of intelligent environmental protection}

"Environmental protection" wisdom is based on the "digital green" platform, on-line monitoring, environmental monitoring network emergency command system, combined with the Internet of things technology, cloud computing technology, 3 s technology, multinetwork integration and other technology solutions, through real-time acquisition of pollution sources, environmental quality and ecological, environmental risks, such as information, build a complete coverage of an omni-directional, multilevel and ecological environment monitoring network, Efficient, accurate transmission environment information resources and huge amounts of data resource center and unified service 
support platform construction, attention to resources reorganization and optimization of dynamic components and application of the application platform, with a more detailed and dynamic way to achieve environmental management and decision of "wisdom", so as to build "inter-operability perception measure more thoroughly, more reliable, Intelligent application more in-depth "intelligent environmental protection" Internet of Things system, to achieve the intelligent environmental protection.

\section{The present situation and existing problems of environmental governance in Liaoning Province}

\subsection{The present situation of environmental management in liaoning province}

Based on the questionnaire survey and field investigation on the government units and enterprises related to urban environmental governance in Liaoning Province, the current environmental governance situation in Liaoning Province is poor, and the governance situation is shown as follows:

Regional development is still uneven. In Liaoning Province, the distribution of infrastructure is unbalanced and the level of economic development is different. Therefore, there are obvious regional differences in environmental governance. In the areas guided by government policies and with better governance institutions, the level of urban environmental governance is higher. In the cities with imperfect infrastructure and relatively slow development, the importance and development level of environmental governance are relatively low.

Information sharing has not yet taken place. The data related to environmental governance in Liaoning Province cannot be transmitted and intercommunicated horizontally and vertically, and there is a lack of sharing mechanism among various departments, resulting in the problem of "information island". The vertical data sharing between the governance subject and the grassroots governance unit is inconsistent and the information conversion is difficult. There are also problems with horizontal data sharing among related units. In the context of smart environmental governance, a good data interconnection mechanism cannot be established for environmental governance, resulting in a decline in the efficiency of environmental governance.

Departmental synergy lacks long-term mechanism. Environmental governance departments in Liaoning Province lack of reasonable and effective collaborative governance mechanism, and the efficiency of environmental governance is low. The processing procedure of environmental government affairs is complicated, and each department can only exist the phenomenon of overlapping and unclear functions. The degree of integration of environmental governance and digital technology is low, and it is difficult to achieve intelligent governance. For example, problems in environmental planning, design and construction have not been solved, and traditional methods and procedures are still continued in the management work, which fundamentally increases the difficulty of intelligent environmental governance.

The supporting system of urban environmental governance is not perfect. Although many cities in the province have applied intelligent governance methods, they have not taken them as the main methods of urban governance. In terms of environmental governance, the relevant supporting systems are not perfect. The technical level of environmental governance needs to be improved, and there is a lack of multi-functional, integrated and all-covering intelligent environmental protection system as the supporting point of environmental governance. There are problems in post setting and personnel selection. The post setting of environmental department is chaotic, and the technical ability of management personnel is low. The effective application of intelligent environmental protection system cannot be separated from a sound system guarantee and an accurate assessment of environmental quality issues. The environmental protection department of Liaoning Province should pay attention to the improvement of relevant systems to ensure the smooth progress of environmental governance process.

\subsection{Problems exist in environmental governance in Liaoning Province}

According to the current situation of environmental governance in Liaoning Province, the following common problems exist in environmental governance in Liaoning Province:

Liaoning Province's urban environmental monitoring capacity is generally insufficient, and the environmental monitoring institutions are overburdened with internal monitoring tasks. The workload of monitoring institutions is heavy, and the gap of technical monitoring personnel is large. The selection and recruitment of monitoring professionals, emphasis on examination rather than technology, monitoring level is uneven, can not be competent for complex monitoring work.

Lack of cooperation among urban environmental governance subjects in Liaoning Province. For example, the interaction between government departments and enterprises and institutions related to environmental governance is less, and the social and public participation is not high. In the face of environmental governance problems, most of them require the cooperation of the society, the government and the public in order to solve environmental problems efficiently and timely. Therefore, a platform and mechanism of tripartite cooperation should be established in intelligent environmental protection.

Due to the lack of long-term coordination mechanism among various departments and the lack of information sharing, environmental resource data is not centralized. Moreover, there are problems such as overstaffed environmental governance institutions, overlapping work 
and unclear functions, which lead to low efficiency of environmental governance.

\section{Intelligent environmental protection system platform}

In view of the above analysis of environmental governance problems in Liaoning Province, under the background of intelligent environmental protection, an intelligent environmental protection service platform is constructed to improve and alleviate the existing problems in urban environmental governance in Liaoning Province through intelligent monitoring and early warning system, intelligent environmental protection supervision system and intelligent waste trading system. For example, through the establishment of an intelligent environmental monitoring and early warning system, the environmental monitoring capacity of Liaoning Province can be greatly improved, and the efficiency of monitoring work can be improved. Through the development of waste intelligent trading system, enterprises can participate in environmental governance, reduce environmental pollution through waste recycling, and enhance the environmental awareness of enterprises. To realize the mode of government, enterprises and citizens co-governance, solve the lack of cooperation between the main bodies of urban environmental governance in Liaoning Province; Through the construction of an intelligent environmental protection system, the environmental governance institutions will be flattened, the data of environmental resources will be integrated, and the public will be facilitated to understand environmental information. We should solve problems such as lack of centralized data on environmental resources, overstaffed environmental monitoring agencies, overlapping work and unclear functions.

Environmental Data Resource Centre

Through the collection and integration of information resources related to environmental governance, data support will be provided for the follow-up service application. Environmental governance involves water resources conservation, air pollution prevention and control, soil remediation, natural ecological protection, conservation and intensive use of environmental space, real-time update of meteorological data and other aspects. Environmental data resource centre provides basic and comprehensive data for complex and diversified environmental governance business.
Intelligent

environmental

awareness

layer/communication layer

Data fusion is realized through perception unit, network transmission and data processing links to form intelligent information conducive to environmental governance. The sensing unit includes sensor, camera, radar, etc. Transmit data through the environmental protection Internet of Things, private network, Intranet, Internet, mobile network, etc. Data processing procedures include data pre-processing, data fusion, abnormal data identification, data quality assurance, etc.

Intelligent environmental protection application layer

We will establish an intelligent monitoring and early warning system. Through the whole process system of monitoring and warning in advance, real-time monitoring in the process and auxiliary rescue after the event, the accurate docking of environmental governance issues is realized. In the pre-monitoring and early warning, the system mainly carries out the real-time dynamic perception of data to ensure the timely discovery of environmental problems; Real-time monitoring is realized through information storage and uninterrupted transmission, as well as intelligent data processing, which is the key to deal with environmental problems, so that early warning mechanism and countermeasures can be developed quickly and accurately, and aid rescue can be realized after the event.

An intelligent environmental monitoring system will be established. The two-way interaction between the public and the government can be realized through the supervision interactive platform: the public can report environmental problems in the city by taking photos and leaving messages, etc., and the relevant government departments can receive the information from the public through the supervision interactive platform, formulate solutions, and feedback the solutions to the public through the platform.

Waste intelligent service system. At present, one of the prominent problems of urban environmental governance is the pollution of large amount of domestic garbage. Waste recycling can effectively solve the unnecessary waste, and the public as a waste of both producers and consumers, through the waste trading platform of GIS system, precise measuring product demand and realize intelligent acquisition, platform and purchasing information from the product information by demand intelligence screening and matching the corresponding public or enterprise, realize the data transmission, So that the waste to achieve recovery or replacement. 


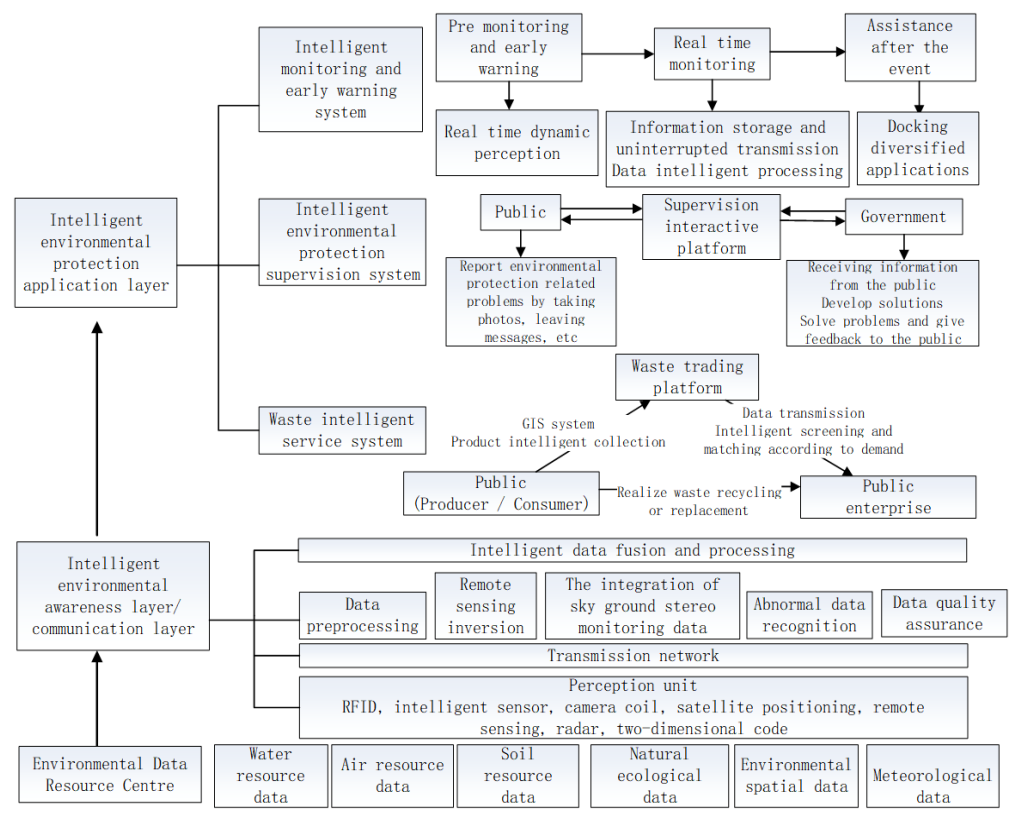

Fig. 1. Intelligent environmental protection system platform

\section{Countermeasures and Suggestions}

\subsection{The main content}

The present situation of urban environmental protection governance modernization in Liaoning Province and other areas in China was investigated. Through field research and interviews, the status quo of urban environmental protection governance in Liaoning Province and the leading cities were investigated, and literature research was adopted to make a comparative analysis of the status quo of urban environmental governance modernization in Hangzhou, Shanghai, Zhengzhou, Foshan, Yantai and other regions.

To analyze the existing problems and causes of urban environmental governance in Liaoning Province. Largescale population gathering in cities brings great pressure to cities, and urban environmental pollution is becoming more and more serious. A series of problems exist in the process of environmental remediation. These problems seriously hinder the improvement of urban governance efficiency, mainly including governance bodies, governance systems, governance concepts, governance methods and other aspects.

To build an intelligent environmental protection service platform in Liaoning Province. We will build an intelligent monitoring and early warning system, an intelligent environmental protection supervision system and an intelligent waste service system, and strengthen cooperation between the government, the market and the general public.

Draw lessons from the experience of other areas and put forward countermeasures and suggestions for the modernization of urban environmental protection management in Liaoning Province. The development and application of urban smart environmental protection in Liaoning Province should be improved and adjusted from several aspects, such as improving the concept, rationally constructing the hardware system, developing and using advanced data processing functions, and improving the law enforcement mode and ability.

\subsection{Countermeasures and Suggestions}

We will further strengthen institutional building. Strengthen the system construction of environmental governance based on the intelligent environmental protection system, clarify the horizontal and vertical function division of the environmental governance department, and improve the internal work efficiency and business level of the department through the division of internal functions and posts and institutionalized management; At the same time, the responsibilities of coordination between environmental protection departments and foreign units are further clarified.

$\mathrm{Re}$ raise the enterprise in the environmental protection aspect's main body consciousness and the consciousness. Enterprises should control pollutants at the source, consciously follow policies and regulations related to environmental protection, actively cooperate with environmental protection authorities, have the courage to assume corresponding responsibilities, and actively accept social supervision.

Further strengthen the intelligent environmental protection system upgrade, expand the application of data fusion. Through information and data sharing and data integration at the provincial, municipal and district (county) levels, the accuracy and stability of early warning, supervision and monitoring of environmental governance will be improved.

We will further promote interconnection and information sharing among environmental protection departments. Through the smart environmental protection system, environmental protection related data will be integrated to alleviate the problem of "information island", effectively improve the efficiency and quality of environmental governance, and promote 
the improvement of the modernization level of urban governance in Liaoning Province.

\section{Author}

${ }^{*}$ Corresponding author: Professor, master supervisor. She is an expert of entrepreneurship education in Liaoning Province and a senior trainer of "KAB Entrepreneurship Education (China) project" of international labor organization.qiankundl@163.com

\section{Acknowledgments}

This paper is the research achievement of Liaoning social science planning fund project. (Project number:L20BGL042)

\section{References}

1. L. Rui, L, Wen qing et al. Research on the development of "Internet +" intelligent environmental protection technology. Engineering Science, J.22,4, (2020)

2. Z. Juntao, T. Rui. Research on Urban Environmental Governance Efficiency and Its Influencing Factors.Research on Finance and Economics,J.6,(2019)

3. C. Zui et al. Intelligent prediction of complex risks of urban lifeline based on big data application.Socialism Research,J.3,(2020)

4. F.Xiaolei, L.Huaizhong et al.Engineering application of intelligent geological hazard monitoring and early warning system based on the whole business chain of "perception, transmission, intelligence and application".Satellite Applications,J.6,(2020)

5. C.Xinghua, M.Haiqiang.Construction and practice of ecological environment monitoring and early warning platform.Environment and Development,J.32,7,(2020)

6. W.Zilong. Thinking on the platform structure of the new type of waste and waste recycling. Journal of Environmental Science, J.35,3,(2016)

7. W.Jianwei. Research on the Application of Smart Environmental Protection in Urban Environmental Governance. Environment and Development, J.31,7,(2019)

8. Y.Xin, L.Rui et al. Theory and Practice of Intelligent Environmental Protection.Beijing: Science Press,M.(2014)

9. Z.Cheng. Smart environmental protection creation method of smart city spatiotemporal information cloud platform.Computer Products and Circulation,J.7 (2019)

10. H.Bing. Design of intelligent environmental protection equipment operation monitoring system based on Web. Energy Conservation and Environmental Protection, J.(2019) 